\title{
Le problème de la preuve et ses effets sur la constitution de la sociologie
}

\section{Philippe Steiner}

\section{OpenEdition}

\section{Journals}

Édition électronique

URL : http://journals.openedition.org/ress/384

DOI : 10.4000/ress.384

ISSN : 1663-4446

Éditeur

Librairie Droz

Édition imprimée

Date de publication : 10 décembre 2003

Pagination : 93-106

ISBN : 2-600-00913-2

ISSN : 0048-8046

Référence électronique

Philippe Steiner, «Le problème de la preuve et ses effets sur la constitution de la sociologie », Revue européenne des sciences sociales [En ligne], XLI-128 | 2003, mis en ligne le 11 novembre 2009, consulté le 21 avril 2019. URL : http://journals.openedition.org/ress/384 ; DOI : 10.4000/ress.384 
Philippe STEINER

\section{LE PROBLÈME DE LA PREUVE ET SES EFFETS SUR LA CONSTITUTION DE LA SOCIOLOGIE}

SOMMAIRE: 1. La position de Durkheim en matière d'administration de la preuve. - 2. Les durkheimiens face à l'absence de preuve: le cas de Weber. 3. L'administration de la preuve: conflits sur la nature de la sociologie et de son institutionnalisation. -4 . Conclusion.

Le propos de ce texte est d'inscrire la question de la preuve en sociologie dans le contexte de la création (au sens de son inscription au sein du cursus universitaire) d'une nouvelle discipline à l'intérieur des sciences sociales. Dans ce contexte, se font jour les propositions d'Emile Durkheim - ainsi que de ses disciples comme Marcel Mauss et François Simiand - et de Max Weber, deux sociologues considérés de nos jours comme caractéristiques de deux manières différentes de faire de la sociologie. Ce contexte constitue une épreuve paradigmatique tant dans la période dans laquelle elle se déroule historiquement et pour les acteurs qui y sont engagés, que pour ceux qui s'y intéressent jusqu'à nos jours. Ainsi, le contexte peut servir de pierre de touche à la réflexion engageant des notions tirées de la sociologie pragmatique contemporaine en termes d'épreuve et de solution de celle-ci par l'intermédiaire d'une procédure d'accord sur une forme de bien commun: une science de la société dont l'élaboration, chez Durkheim comme chez Weber, comporte une dimension morale ne serait-ce que par la «clarté» qu'elle exige de la part de ceux qui s'en inspirent (Weber 1919: 101-104)

\section{LA POSITION DE DURKHEIM EN MATIÈRE D'ADMINISTRATION DE LA PREUVE}

Dans son ouvrage bien connu sur la méthode sociologique, ouvrage controversé de sa première parution à nos jours ${ }^{1}$, Durkheim suit une progression très maîtrisée - comme l'objet de son texte le commande d'ailleurs - établissant les règles à suivre en matière de la définition, de l'observation et de la classification du social. Cette première partie de l'exposé amène à deux chapitres clés concernant successivement les règles à suivre dans l'explication sociologique et celles relatives à l'administration de la preuve, chapitre sur lequel se termine l'ouvrage, avant une intéressante conclusion qui fait plus que résumer le contenu de l'exposé. On y reviendra.

\footnotetext{
On peut en prendre la mesure au travers de deux recueils (Borlandi \& Mucchielli 1995; Cuin
} 1996) édités à l'occasion du centenaire de l'ouvrage. 
Le point intéressant pour ce qui nous concerne ici tient dans l'articulation entre les deux chapitres consacrés à l'explication et à l'administration de la preuve. L'origine d'une telle division de la réflexion de Durkheim se trouve très probablement dans la manière dont Claude Bernard a présenté le raisonnement expérimental en médecine (Bernard 1865) et que Durkheim suit comme un modèle dans son propre exposé (Berthelot 1988). En effet, Bernard est soucieux de distinguer la simple coexistence ou simple succession dans le temps de deux phénomènes car ces deux cas de figure peuvent laisser croire à l'observateur inattentif qu'il existe une «loi » là où il n'y en a pas, c'est-à-dire là où il n'y a pas de relation de causalité entre les phénomènes considérés. En conséquence, Bernard impose ce qu'il appelle une contre-preuve ou contre-expérimentation: le fait à expliquer se trouve-t-il présent lorsque ce que l'on tient comme sa cause n'est plus présent?

Au-delà de cette référence à une source - sinon la source - de la réflexion de Durkheim, la justification de ces deux chapitres tient au fait que Durkheim insiste sur une forme d'explication sociologique spécifique, au moins dans la période dans laquelle il écrit. Le fait social est un fait sui generis, dit Durkheim pour s'opposer à toute démarche réductionniste ${ }^{2}$, démarche qu'il critique en prenant pour cible l'économie politique ou le cartésianisme qui participent tous les deux du rationalisme classique. Dans le cadre d'une science expérimentale le fait social est expliqué par sa cause efficiente et non pas par ce à quoi il sert ou par la finalité qu'ont en vue les acteurs, surtout lorsque celle-ci est constatée après les faits. Mettant le social face au social, s'interdisant de ramener le social à ses composantes et à trouver l'explication du social dans autre chose que lui-même (la psychologie individuelle ou la biologie), Durkheim se trouve contraint de clarifier les conditions de validité de l'explication. Comment prouve-t-on que l'explication est acceptable? On connaît la réponse: la preuve est administrée en s'appuyant sur ce que John Stuart Mill a dénommé la méthode des variations concomitantes. Cette réponse ouvre, mais ce n'est en rien systématique ${ }^{3}$, sur les procédures statistiques de la preuve et, dans le cas de Durkheim, sur des techniques du genre de celle de la corrélation statistique entre des séries temporelles dans un modèle multivarié, même si Durkheim n'a jamais mis en œuvre lui-même une telle technique, trop récente dans son emploi en sciences sociales puisque les articles de Karl Pearson et de Udni Yule paraissent en 1897 (Westergaard 1932: $150,271-2)$. On en a un exemple frappant lorsque Durkheim présente son étude de la relation entre suicide et statut matrimonial en énonçant quatre lois à partir de données statistiques françaises et allemandes sur les taux de suicide et le calcul des coefficients de préservation ${ }^{4}$, et qu'il indique: «Les faits étant ainsi établis, il

Au sens où le phénomène social à expliquer serait réduit à ses composants - les acteurs euxmêmes - et cette réduction est le gage d'une explication correcte, c'est-à-dire qu'elle permet d'administrer la preuve de l'explication.

Une telle procédure statistique est certes à l'œuvre dans le Suicide (Durkheim 1897), mais tel n'est pas le cas dans La division du travail et encore moins dans les Formes élémentaires, ouvrage consacré à une seule «expérience», celle fournie par les Aruntas.

«Les lois qui se dégagent de ces tableaux peuvent se formuler ainsi: $1^{\circ}$ Les mariages trop précoces ont une influence aggravante sur le suicide, surtout en ce qui concerne les hommes [...] $2^{\circ}$ A partir de 20 ans, les mariés des deux sexes bénéficient d'un coefficient de préservation par 
nous faut chercher à les expliquer» (Durkheim 1897: 185). L'explication passe alors par une discussion fine des données statistiques interprétées selon la théorie de la socialisation qui est la base théorique de l'auteur (Besnard 1987).

On a remarqué à de nombreuses reprises que le traitement des données laissait parfois à désirer chez Durkheim (Pope 1976, Besnard 1987), néanmoins, et contre l'avis de Vilfredo Pareto ${ }^{5}$, le travail de Durkheim est robuste: il peut donner lieu à rectification sans que cela mette en cause la généralité des résultats acquis, et il peut être prolongé jusqu'à nos jours sur les données récentes interprétées avec des techniques statistiques plus élaborées (Halbwachs 1930, Baudelot \& Establet 1984).

Les durkheimiens vont largement se ranger autour de cette procédure, mais non sans y introduire des variations importantes. Simiand va prendre au pied de la lettre la démarche durkheimienne pour élaborer ses travaux de sociologie économique, tous nourris d'une documentation statistique très lourde, méticuleusement critiquée (au sens de la critique historienne des sources) ${ }^{6}$. Cette démarche est présentée lors de l'allocution que Simiand prononce à titre de président de la Société de statistique de Paris en 1921 sous le titre de «La statistique comme moyen d'expérimentation et de preuve» (Simiand 1922: ii); la notion de preuve y occupe une place centrale comme on le lit dans l'avant-propos:

Telles quelles, si ces remarques peuvent contribuer à préciser la notion même de statistique et à la lier, dans un juste rapport, avec celle d'expérience, puis à donner conscience des conditions à envisager pour un emploi probant de statistiques et suggérer en même temps les moyens d'y satisfaire, cette publication aura atteint tout le but auquel elle pouvait viser (ibid: iii).

rapport aux célibataires [...] $3^{\circ}$ Le coefficient de préservation des mariés par rapport aux célibataires varie avec les sexes [...] $4^{\circ}$ Le veuvage diminue le coefficient des époux des deux sexes, mais, le plus souvent, il ne le supprime pas complètement» (Durkheim 1897: 182-5). Rappelons qu'un coefficient de préservation est le rapport de deux taux de suicide; par exemple, le coefficient de préservation des mariés sur les célibataires, pour les hommes d'une tranche d'âge donnée, est égal au rapport entre le taux de suicide des célibataires et celui des mariés.

s. Dans un compte rendu du Suicide paru dans le Zeitschrift für Sozialwissenschaft, Pareto porte un jugement sévère sur le travail de Durkheim: «Le raisonnement est malheureusement, dans tout l'ouvrage, fort peu rigoureux. C'est là d'ailleurs un caractère que l'on rencontre dans un grand nombre d'ouvrages sociologiques de notre époque. Les auteurs discutent fort peu les sources de leurs informations et donnent souvent des rapprochements fortuits pour des démonstrations rigoureuses. Pour noter toutes les erreurs de ce genre dans l'ouvrage que nous examinons, il faudrait écrire un autre ouvrage d'un nombre égal, ou même plus grand de pages; nous nous bornerons à citer quelques exemples» (Pareto 1898: 122). En fait, Pareto concentre sa critique sur les données figurant en introduction, données que Durkheim commente avec quelque partialité, nous y avons insisté ailleurs (Steiner 2000: 64-5, 81).

6 On peut en prendre la mesure en examinant l'ouvrage issue de la thèse de droit que Simiand fait paraître en 1907 et, plus encore, dans le grand ouvrage de la maturité (Simiand 1932) lequel comporte un volume (le premier) entièrement consacré à l'explicitation méthodologique de l'usage des données quantitatives en économie positive et un autre (le troisième) entièrement consacré à la présentation des données (tableaux et graphiques) et à la critique de celles-ci. Sur l'activité institutionnelle de Simiand en matière de statistiques il convient de se reporter à l'ouvrage d'Alain Desrosières (1993). 
La réflexion de Simiand l'amène à préconiser un travail serré sur la nature des mesures - quel est le phénomène collectif qui se trouve derrière et comment l'apprécier quantitativement - à privilégier les grandeurs relatives plutôt qu'absolues, car on élimine les erreurs constantes et on peut suivre «le phénomène se produisant» (ibid: 39) et à imposer une multiplicité d'expériences statistiques de manière à déboucher sur des comparaisons sans lesquelles le chercheur reste prisonniers de la qualité (parfois médiocre) de ses données (ibid: 52). L'importance attribuée à la statistique ne donne pas lieu à un traitement technique des données recueillies au-delà d'un calcul de moyennes; Simiand laisse dans le flou la dimension purement technique de la statistique - qu'il considère d'ailleurs comme non aboutie à ce jour dans le domaine de l'économie politique (ibid: 60 61 ) - et ne met jamais en œuvre le calcul des coefficients de corrélation. A notre connaissance, le seul à s'intéresser de près à cette technique chez les durkheimiens est Maurice Halbwachs dont il faut noter la collaboration avec Maurice Fréchet, mathématicien rencontré à l'Université de Strasbourg, pour la rédaction d'un ouvrage d'introduction aux techniques statistiques (Fréchet \& Halbwachs 1924) ${ }^{7}$. Il n'en reste pas moins que les travaux durkheimiens qui administrent la preuve de leur explication sociologique au moyen de données de nature quantitative ne sont pas très nombreux. A côté de Simiand et de Halbwachs, on peut placer quelques travaux de Hubert Bourgin, l'article que Mauss rédige avec Henri Beuchat sur les Esquimos, mais ni Célestin Bouglé, ni Paul Fauconnet, ni Paul Lapie ne mettront en œuvre une telle technique d'administration de la preuve.

Les durkheimiens ne sont pas non plus unanimes quant à l'importance à accorder à cette forme d'administration de la preuve. On peut le voir en considérant le long article «Sociologie» que Fauconnet et Mauss publient dans la Grande encyclopédie en 1901, article auquel Durkheim a donné la main et dont il a surveillé de très prés la rédaction ${ }^{8}$. Dans cet article qui suit le fil de l'argumenta-

A cette exception près, qui n'est d'ailleurs pas suivie d'une pratique de la statistique probabiliste par Halbwachs, on peut dire que les durkheimiens s'en tiennent à une conception ancienne de la statistique, laquelle met l'accent sur l'accumulation des matériaux et sur une critique externe de ceux-ci pour déterminer leur qualité. Cette démarche s'oppose à celle qui place son espoir dans une analyse interne des données recueillies, analyse menée au moyen des outils de la statistique probabiliste (Westergaard 1932: 262-4, Stigler 1986: 222, Desrosières 1993: 345-9). Dans le premier cas, on s'oriente vers un usage descriptif des statistiques socio-économiques, alors que le second débouche sur l'économétrie.

8 La correspondance entre Durkheim et Mauss ne laisse aucun doute sur ce point. En avril et mai 1900, Durkheim envoie deux lettres à Mauss à propos de cet article, il leur suggère un plan et propose à deux reprises de collaborer avec eux à la rédaction de ce texte (Durkheim 1998: 257261). Cette dernière proposition ne semble pas avoir eu de suite, mais Durkheim discute de nouveau leur plan, ce mois de mai 1900 (ibid: 265). Passant à Paris en août, il rencontre Fauconnet pour travailler avec lui sur leur manuscrit et il juge médiocre ce qu'a fait Mauss: «J'ai vu Fauconnet à Versailles et nous avons travaillé. Ton brouillon a besoin de réfections graves dans la première partie, les idées sont exposées en dehors de tout ordre didactique, et même de tout ordre; la seconde ('Explication') est beaucoup trop étriquée. Nous avons refait un plan avec Fauconnet qui va l'exécuter en se servant de ce que tu as fait pour la première partie. La seconde est presque tout entièrement à faire. La troisième ('Méthode') se tient mieux » (lettre du 3 août 1900, ibid: 268). Cinq jours plus tard, il revient sur le sujet et indique : «J'ai reçu la première partie du travail de Fauconnet, c'est-à-dire l'Objet refait d'après le plan convenu à Versailles. Je ne l'ai lu encore que rapidement afin de voir seulement l'ensemble; je crois que c'est tout à fait cela. C'est étoffé, 
tion des Règles, les auteurs laissent entièrement de côté cette partie de la méthode exposée quelques années avant par Durkheim; en lieu et place, figure une courte réflexion sur le caractère scientifique des hypothèses proposées par le sociologue, au sens où ces hypothèses sont «éminemment critiquables et vérifiables» (Fauconnet \& Mauss 1901: 172).

Néanmoins, l'emprise de l'administration statistique de la preuve fait son chemin chez Mauss par l'intermédiaire de l'importance croissante que ce dernier accorde à la quantification en sociologie, y compris en anthropologie ou en sociologie religieuse, ce qui explique que les chercheurs les plus productifs et les plus originaux de l'école durkheimienne donnent assez volontiers l'impression de se regrouper derrière la démarche de Durkheim d'administration statistique de la preuve de l'explication sociologique.

\section{LES DURKHEIMIENS \\ FACE À L'ABSENCE DE PREUVE: LE CAS DE WEBER}

On peut bien sûr chercher à opposer la démarche des durkheimiens et celle de leur grand contemporain qu'on leur a souvent fait reproche d'avoir négligé voire purement et simplement ignoré. Ce dernier reproche est entièrement non fondé (Steiner 1992), mais le premier peut avoir un fondement lorsqu'on examine la récurrence des jugements que les durkheimiens portent lorsqu'ils lisent les travaux de Weber.

Il est éclairant de rapprocher quelques jugements parmi ceux qui nous sont parvenus et qui traitent de la question de la méthode. On voit alors l'importance qu'acquiert la notion de preuve comme c'est le cas ici de Halbwachs (1929) dans un article sur l'Ethique protestante et l'esprit du capitalisme, article considéré comme un des rares commentaires ayant compris l'argumentation de Weber. Après une longue présentation, Halbwachs termine par une page de conclusion dont j'extraie la première phrase et le dernier paragraphe:

\footnotetext{
Toute cette étude de Max Weber est un curieux exemple d'une hypothèse vraiment originale et saisissante, mais engagée encore à demi dans une masse de faits. Elle nous aide déjà à en mieux apercevoir le sens. Mais elle nous oblige aussi à explorer plus avant tout ce qu'elle nous en découvre [...] Cette thèse se fortifie donc du témoignage qu'apporte en sa faveur le théologien protestant [Ernst Troeltsch] sans doute le mieux informé, le plus vigoureux et le plus profond d'à présent. Les historiens économistes d'autre part ne paraissent pas connaître mieux, ni même aussi bien que Max Weber toute cette période, en sorte qu'ils ne furent pas en mesure, jusqu'ici, de le suivre sur le terrain où il s'était le premier engagé. Pour toutes ces raisons, il valait la peine d'offrir au lecteur français un aperçu de ces recherches si hypothétiques et provisoires qu'en pussent être encore les résultats » (Halbwachs 1925: 153-154)
}

très clair et de nature, je l'espère, à porter. Je vais maintenant voir le détail. Mais il ne me paraît pas que les retouches nécessaires doivent être très nombreuses ni surtout très difficiles. Je partirai d'ici le 16. Mais le travail utile ne pourra être commencé que quand nous serons tous les trois ensemble, c'est-à-dire quand Fauconnet sera à Epinal» (lettre du 8 août 1900, ibid: 269). 
Quelques années plus tard, Mauss livre un jugement intéressant pour notre propos dans une lettre à Roger Bastide lequel, après avoir envoyé à Mauss son ouvrage sur la sociologie de la religion, lui demande conseil pour rédiger une thèse sur les cadres sociaux de la vie mystique «dans la voie, par conséquent, de certaines de vos pages sur la magie [...] dans la voie, également, des recherches de Max Weber» (lettre à Mauss du 20 octobre 1936) ${ }^{9}$ :

\begin{abstract}
J'ai bien reçu votre petit livre [...]. J'ajoute que l'un de ceux que vous appréciez particulièrement, Max Weber, est un de ceux avec lesquels Durkheim, Hubert et moi nous communions le moins. Naturellement, quand il se contentait de nous démarquer, ce qu'il fit longuement pendant la guerre - époque où tout était possible -, nous avions de quoi nous agacer. Mais lui s'est borné à émettre des opinions dont un grand nombre sont suggestives, et quelques-unes valables, mais dont aucune, sauf exception, n'est prouvée (lettre du 3 novembre 1936)
\end{abstract}

La similitude des jugements de Halbwachs et de Mauss portés en termes de preuve est frappante. Dans les deux cas ${ }^{10}$, les hypothèses dégagées par Weber en ce qui concerne la relation entre religion et capitalisme sont considérées comme suggestives et dignes d'être présentées au lecteur pour leur propre réflexion. Mais elles sont aussi systématiquement jugées comme de simples hypothèses dont aucune n'est prouvée, dit Mauss, dont les résultats ne sont qu'hypothétiques et provisoires, dit Halbwachs dans un langage moins sévère. On ne peut que regretter que Mauss n'ait pas pris le temps d'indiquer précisément celles des opinions de Weber qu'il range dans les exceptions, c'est-à-dire lesquelles des opinions émises par Weber sont, d'après son critère, prouvées.

Néanmoins, il ne faut pas s'arrêter à ce niveau des commentaires, car on courrait le risque d'en rester à un niveau superficiel dans la confrontation entre deux formes de sociologie qu'on a pris l'habitude d'opposer, opposition qui est devenue comme le pont aux ânes de la sociologie académique. Il faut en effet se poser deux questions avant d'aller plus loin: premièrement, quelle est la position de Weber en ce qui concerne la question de la preuve? deuxièmement, Weber n'offre-t-il pas une diversité d'approches sociologiques qui pourrait ressembler, finalement, à ce que nous avons pu trouver chez Durkheim et le groupe des durkheimiens?

A propos de la première question, il est intéressant de se reporter au premier chapitre d'Economie et société - chapitre que Weber avait relu et corrigé pour l'impression avant sa mort - où il présente ce que l'on appelle depuis la sociologie compréhensive. Dans ce cadre, la première étape du travail scientifique est de

Je dois à Roland Lardinois la connaissance de cette correspondance et à Françoise Morin de pouvoir en extraire quelques passages à l'occasion de la rédaction de ce texte-qu'ils trouvent ici l'expression de mes remerciements. La correspondance permet de comprendre pourquoi Mauss donne son avis sur Weber puisque Bastide y fait explicitement allusion comme une des voies qu'il entend poursuivre dans son travail de thèse, alors que cette voie est peu présente dans l'ouvrage où Bastide présente et discute abondamment les positions de Durkheim et des durkheimiens (Bastide 1935).

1o Ici je suppose que le jugement porté par Mauss concerne les études de sociologie religieuse de Weber. La nature de l'ouvrage qu'il a reçu, le fait qu'il mentionne dans un même mouvement Durkheim, Hubert et lui-même dans la période de la guerre - Weber publie ses études de sociologie religieuse (Die Wirtschaftsethik der Weltreligionen) en 1915 - permettent de le penser. 
déterminer le sens de l'action pour l'acteur; Weber est conscient des difficultés que comporte une telle méthode, d'où l'importance accordée par lui aux modalités de contrôle des hypothèses faites sur le sens donné à l'action, aussi évidentes qu'elles puissent paraître d'un point de vue de l'explication causale (Weber 1921: 8-9). Il suggère de dissiper la gêne sur ce point au moyen des procédures suivantes :

Tout comme pour toute autre hypothèse, il est indispensable de contrôler l'interprétation significative compréhensible par le résultat, c'est-à-dire la tournure prise par le déroulement réel de l'activité. On n'y parvient avec une relative exactitude que dans des cas, malheureusement très rares, qui s'y prêtent en raison de leur nature particulière, dans l'expérimentation psychologique. On y arrive aussi avec une approximation extrêmement variable, grâce à la statistique, dans les cas (également limités) de phénomènes collectifs dénombrables et univoques d'un point de vue de leur imputation. Pour le reste il n'existe d'autre possibilité que la comparaison de processus aussi nombreux que possible de la vie quotidienne et journalière qui sont semblables en tout, mais diffèrent en un unique point, celui du 'motif' ou 'facteur' dont l'importance pratique fait chaque fois l'objet de la recherche: c'est là une tâche importante de la sociologie comparée. Lorsque nous voulons faire une imputation causale il ne nous reste malheureusement souvent que le moyen incertain de l'expérience mentale, c'est-à-dire imaginer la suite possible d'événements singuliers de la chaîne des motifs et construire le cours des choses probables en ce cas (ibid: 9).

Sans donner prise à une élaboration aussi développée que celle de Durkheim, il faut souligner les rapprochements forts entre la démarche de Weber et celle du sociologue français. Weber est soucieux de prouver la validité de l'étape compréhensive au moyen d'une évaluation des résultats, c'est-à-dire du déroulement causal de l'action significativement orientée. Cette preuve peut être fournie selon trois modalités ${ }^{11}$, dont les deuxième et troisième sont similaires à celles mises en œuvre par Durkheim: $1^{\circ}$ l'expérimentation psychologique; $2^{\circ}$ les statistiques lorsqu'il s'agit de phénomènes collectifs; $3^{\circ}$ la méthode comparative, mais pas nécessairement celle des variations concomitantes, puisque Weber fait référence à la méthode des différences. L'exemple que Weber donne de la preuve d'une loi par l'intermédiaire des statistiques est très clair sur ce qu'il entend faire sous la deuxième modalité :

La prétendue 'loi de Gresham' [selon laquelle 'la mauvaise monnaie chasse la bonne'], est une interprétation rationnellement évidente de l'activité humaine dans des conditions données et sous la présupposition idéaltypique d'une activité purement rationnelle en finalité. Pour savoir dans quelle mesure on agit réellement en conformité avec cette loi, il n'y a que l'expérience (qui finalement doit en principe s'exprimer d'une manière ou d'une autre sous une forme 'statistique') qui puisse nous renseigner sur la disparition effective de la circulation des espèces de monnaie dont l'évaluation est chaque fois trop inférieure par rapport au système monétaire: de fait, elle confirme la validité considérable de cette loi (ibid: $9-10$ ).

Il est important de marquer de surcroît que, faisant la différence entre l'ordre de l'exposition et l'ordre de la découverte, Weber se place dans une séquence

La référence à l'expérience mentale ne nous paraît pas placée par Weber au même niveau que les trois premières formes de contrôle méthodologique. C'est un exercice de cohérence logique plutôt qu'une manière de prouver la validité de l'imputation causale. 
intellectuelle dans laquelle il n'y a pas de différence significative avec Durkheim lorsque ce dernier explicitait le lien entre l'approche expérimentale (repérage des régularité puis explication sociologique de celles-ci) et l'administration de la preuve:

En vérité, le cheminement de la connaissance a été le suivant: les observations d'ordre expérimental ont précédé l'interprétation qui en a permis la formulation. Sans cette interprétation réussie, notre besoin causal serait manifestement insatisfait. Sans la confirmation par preuve, en outre, que le développement effectivement intervenu, dans une mesure quelconque, une telle loi, si évidente qu'elle puisse être en soi ne constituerait pour la connaissance de l'activité effective qu'une connaissance sans valeur. Dans cet exemple, la concordance entre l'adéquation significative et la vérification par l'expérience est absolument concluante et les cas sont suffisamment nombreux pour qu'on admette que la preuve est suffisamment établie (ibid: 10).

Le même type de rapprochement peut être fait à propos de la deuxième procédure évoquée par Weber, la comparaison. On peut d'abord rappeler combien Durkheim appréciait une telle approche quand on sait que, pour lui, en sociologie, expliquer c'est comparer (Durkheim 1897: 1). En outre, on peut mentionner, pour en rester au domaine à la croisée de la sociologie religieuse et de la sociologie économique, domaine dans lequel les intérêts de recherche de Weber sont proches de ceux des durkheimiens, que la procédure suivie par Mauss dans l'Essai sur le don, ouvrage dans lequel il compare les modalités de ce qu'il appelle l'échangedon dans différentes aires culturelles de manière à en tirer des conclusions pour le présent (notamment en ce qui concerne la dimension économique et la prédisposition au calcul du comportement humain en Occident), n'est pas fondamentalement différente de celle suivie par Weber dans sa sociologie de l'éthique économique des religieuses mondiales. Weber cherche à isoler un facteur explicatif du présent (un de ceux qui peuvent expliquer les raisons du développement du rationalisme pratique économique en Occident) alors que Mauss recherche une structure de transfert des biens et des richesses présente dans les différentes aires et sous diverses formes à côté de l'échange marchand, mais leur proximité est forte dès lors que l'on remarque que les deux auteurs mettent en œuvre une approche fondée sur la recherche et la comparaison de dispositifs sociaux spécifiques $^{12}$ : la confirmation (devant son Dieu ou devant les membres d'une secte) menant à un style de vie spécifique (ascétisme intramondain) dans le cas de Weber, les trois obligations de donner-recevoir-rendre suscitant un style de vie en faveur de la «dépense noble», de «l'altruisme» dans le cas de Mauss. En faisant cette remarque je veux insister sur le fait que la mise en regard de ces deux exemples - particulièrement célèbres, parce que particulièrement réussis et productifs - d'application de la méthode comparative en sociologie, repose sur un même socle méthodologique avec l'existence d'un dispositif social, c'est-à-dire d'une configuration dans laquelle les relations sociales sont agencées de manière à produire des régularités jugées significatives et observables par le sociologue ${ }^{13}$.

12 Ce terme de dispositif social est très proche de celui de «mécanisme générateur» (Cherkaoui 1997) ou de «mécanisme social» (Hedström \& Swedberg 1998).

13 On pourrait rajouter le fait que Durkheim (1904-5) lui-même s'avance dans une voie similaire lorsqu'il dégage le mécanisme de l'affirmation et de la confirmation de la valeur scolaire en 
Finalement, la façon dont Weber procède dans ses recherches - et non pas dans ses textes consacrés à la méthode - se coule assez facilement dans le cadre de ce qui vient d'être explicité à propos du premier chapitre d'Economie et société. Comme Halbwachs n'a pas manqué de le signaler dans son étude, Weber mentionne les recherches d'un de ses étudiants - Martin Offenbacher - sur la relation entre confession religieuse et type de scolarisation et il en présente l'argument au travers de données statistiques. De même, Weber a fait un important travail de type quantitatif dans son étude sur la paysannerie Est-elbienne ou sur l'industrie allemande (Weber 1908), même si l'on peut dire avec les commentateurs de cette partie moins visitée de son œuvre qu'il n'en a pas retiré de résultats bien nets au moins pour ce qui concerne la dernière d'entre elle.

Bref, les modes de travail dans ces deux approches de la sociologie diffèrent bien moins en pratique que ce que l'on en dit maintenant, y compris à propos de la question de l'administration de la preuve. Cela ne veut-il pas dire que cette question de la preuve ressortit essentiellement à l'épreuve, c'est-à-dire à des modalités de résolution de la polémique scientifique, et qu'elle revêt une dimension plus rhétorique que méthodologique ou logique?

\section{L'ADMINISTRATION DE LA PREUVE: CONFLITS SUR LA NATURE DE LA SOCIOLOGIE ET DE SON INSTITUTIONNALISATION}

Les passages de Mauss et de Halbwachs cités plus haut contiennent une dimension renvoyant au caractère conflictuel de la communauté scientifique. Mauss fait explicitement référence au fait que Weber se serait inspiré des travaux des durkheimiens sans payer ses dettes à leur égard, d'où l'irritation que lui-même, Hubert (son «frère de recherche») et Durkheim auraient eu vis-à-vis de son œuvre. La dimension polémique est évidente et l'affirmation que les travaux de Weber soient suggestifs, mais non prouvés, achève la démonstration implicite de la supériorité théorique - et morale - des durkheimiens. De son côté, Halbwachs relève la dimension polémique qui est attachée à la recherche de Weber sur le protestantisme: en terminant par une référence aux critiques des historiens économiques et leur incapacité à suivre Weber sur le domaine, Halbwachs fait voir le caractère conflictuel de la construction de la sociologie au sein des sciences sociales allemandes ${ }^{14}$ et la

comparant les formes de pédagogie depuis la Renaissance de manière à comprendre le succès pédagogique des Jésuites. Ce mécanisme se trouve aussi à l'œuvre dans l'étude de Pierre Bourdieu (1979) sur les classes préparatoires françaises et, plus largement, sur les élites dont la formation passe par une phase de clostration scolaire. Nous avons développé ce point par ailleurs (Steiner 2003: chap. 8).

14 Cette dimension est bien connue ne serait-ce que par l'importance des corrections que Weber introduit dans son texte et les longues réponses qu'il fait à ses critiques (Weber 1907-10); dans ce dernier cas, on voit clairement comment son approche de la sociologie compréhensive modifie le régime du discours socio-histortique en introduisant d'autres critères de preuve et de modalités de l'administration de celle-ci. L'article récent de Peter Ghosh (2003) va d'ailleurs pleinement dans ce sens en montrant le déplacement que Weber opère en sélectionnant une série de sources qui n'était pas mobilisées ou pas avec la même intensité par ceux qui s'intéressaient à la science des religions en Allemagne, Troeltsch inclu. 
place éminente que Weber y occupe puisque s'il est question d'hypothèses qui «restent encore à demi engagées dans les faits » et de résultats «hypothétiques et provisoires », il n'en demeure pas moins que les historiens professionnels ne sont pas capables d'en faire une critique pertinente ou d'en proposer des contreépreuves recevables.

Les similitudes existant entre l'approche de Weber et celle de Durkheim et des durkheimiens ne portent pas à donner crédit à l'affirmation de Mauss, affirmation donnée dans une correspondance privée, laquelle ne doit pas faire oublier les jugements plus favorables présents dans des textes antérieurs du même Mauss ${ }^{15}$ puisqu'il fait état d'une communauté de vue entre les deux approches pour les distinguer de ce qu'il caractérise comme du «journalisme rationnel» ou de la «philosophie paresseuse». Ce que l'on retrouve dans cette curieuse interprétation de la sociologie compréhensive de Weber que Mauss donne dans un article de 1934 lorsqu'il s’agit de situer la place des spéculations générales en sociologie:

\begin{abstract}
Nous y tendons à expliquer: comment on peut aborder ces faits, comment on peut les diviser, les énumérer d'une façon suffisamment complète, et enfin, à l'intérieur de ces divisions, les caser très nombreux, bien définis. Ici, nous n'énonçons pas seulement des idées, nous voulons entamer des recherches, conduire à bien des analyses de réalités sociales dont on pourra, un jour, peut-être tout de suite, dégager une théorie. Va et vient nécessaire, car la théorie, si elle est extraite des faits, peut à son tour permettre de les faire voir, de les mieux connaître et de les comprendre. C'est ce que Max Weber appelait la verstehende Soziologie (Mauss 1934: 304)
\end{abstract}

On retrouve alors au cœur de la réflexion de Mauss la question de l'épreuve de force entre la sociologie qui se propose d'accéder au rang de science de la société et les discours sur ce même objet qui ne sont rien d'autre qu'une forme, mise au goût du jour, de moralisme ou bien qui ne vise rien d'autre que l'action. De ce point de vue, la méthode et donc la preuve sont pour Mauss comme pour Durkheim, et sans doute aussi pour Weber, le moyen de faire le départ entre les deux domaines, pour éviter de subir l'empiétement non désiré du discours de sens commun déguisé sous les apparences de la science.

Plus qu'une véritable opposition entre les auteurs considérés, l'opposition se trouve déplacée entre leur projet de création de la sociologie - ou plus exactement de la sociologie à l'intérieur des sciences sociales - et ceux qui ne veulent pas se plier aux exigences de ce type de démarche. La conclusion des Règles de la méthode sociologique est de ce point de vue on ne peut plus claire. Durkheim

15 Dans un de ses textes, Mauss range Weber aux côtés des durkheimiens lorsqu'il s'agit de refuser le morcellement du champ des sciences sociales et de maintenir, au sein de la sociologie, une ambition générale et une pratique de discussion avec les autres sciences sociales, de manière à ne pas régresser au stade de l'anomie (au sens de contacts trop faibles entre des parties d'un ensemble) que redoutaient Auguste Comte comme Durkheim: «Le regretté Max Weber, s'il n'a guère cité Durkheim et l'œuvre faite sous la direction de celui-ci, était beaucoup plus près de notre point de vue. L'état actuel des choses [dans la sociologie allemande] est presque en régression par rapport à cet auteur» (Mauss 1927: 291). L'abandon du dialogue entre les sociologues et les autres sciences sociales se traduit, selon son tour d'horizon des sociologies nationales, par « une sorte de morale compliquée d'un peu de statistique» (ibid: 292) ou par une pure rhétorique brillante, une prédication morale voire religieuse; cette appréciation est la base dont découle la critique de la sociologie en termes de «journalisme rationnel [ou] de philosophie paresseuse» (ibid). 
indique que la sociologie n'est ni une philosophie, ni une prise de position politique (individualisme, socialisme, communisme), bien que les problèmes pratiques ne lui soient pas étrangère (Durkheim 1894: 139-141). Sa démarche est explicitement menée dans le but «d'instituer cette discipline», ce qu'il exprime en termes de preuve:

\begin{abstract}
Non seulement les explications sont autres [que dans les discours de sens commun], mais elles sont autrement démontrées, ou plutôt c'est alors que l'on sent le besoin de les démontrer. Si les phénomènes sociologiques ne sont que des systèmes d'idées objectivées, les expliquer, c'est les repenser dans leur ordre logique et cette explication est à elle-même sa propre preuve; tout au plus peut-il y avoir lieu de la confirmer par quelques exemples. Au contraire, il n'y a que des expériences méthodiques qui puissent arracher leur secret aux choses (ibid: 142).
\end{abstract}

C'est là le moyen d'éviter que la sociologie ne soit qu'une mode, d'éviter que les sociologues s'installent dans l'arène médiatique, visant aux succès mondains que cette dernière leur promet trop facilement:

\begin{abstract}
Nous croyons au contraire que le moment est venu pour la sociologie de renoncer aux succès mondains, pour ainsi parler, et de prendre le caractère ésotérique qui convient à toute science. Elle gagnera ainsi en dignité et en autorité ce qu'elle perdra peut-être en popularité. Car tant qu'elle reste mêlée aux luttes de partis, tant qu'elle se contente d'élaborer, avec plus de logique que le vulgaire, les idées communes et que, par suite, elle ne suppose aucune compétence spéciale, elle n'est pas en droit de parler assez haut pour faire taire les passions et les préjugés (ibid: 144).
\end{abstract}

Une appréciation partiale de ce passage et de nombreux autres pourrait vouloir attribuer au «positivisme» durkheimien, à une survalorisation de la compétence et des techniques «qui font science», les raisons d'une telle prise de position. Ce n'est pas aussi simple. Weber que l'on n'a pas l'habitude d'accabler sous de telles remarques n'est pas éloigné d'une telle prise de position et la raison en est toujours la même: dans les sciences sociales, le spécialiste a toujours à redouter le dilettante qui prend de haut les efforts du spécialiste. Weber présente ainsi les choses lorsqu'il s'explique devant les étudiants berlinois sur la vocation de savant et la mise à distance du dilettante ${ }^{16}$, précisément parce que celui-ci ne s'attache pas à fournir la preuve de la validité de ses intuitions:

L'idée d'un dilettante peut avoir d'un point de vue scientifique exactement la même portée, ou une portée plus grande, que celle du spécialiste. Nous sommes redevables à des dilettantes, justement, de beaucoup de nos meilleurs questionnements et de nos meilleures connaissances. Le dilettante se distingue du spécialiste - comme Helmholtz l'a dit de Robert Mayer - seulement en cela qu'il lui la solide garantie de la méthode de travail et qu'il n'est par conséquent pas en état, la plupart du temps, de contrôler après coup la portée de l'idée et de l'évaluer ou de la mener à son terme» (Weber 1919: 78).

Plus encore, on peut se reporter aux articles avec lesquels Weber répond aux détracteurs de l'Ethique protestante pour voir combien Weber était lui aussi capable de faire valoir les droits du spécialiste quant à ce que Durkheim appelait l'administration de la preuve pour voir que, sur ce point, ils faisaient cause commune. Dans les Anti-critiques il est fréquemment question de la preuve et des

${ }^{16}$ Ce que Durkheim avait fait dès l'introduction à La division du travail. 
moyens de la fournir dans le cas considéré par lui, de manière délibérée. Weber n'arrête pas d'opposer les efforts faits par lui pour «prouver que le concept de Beruf, lequel, avec sa connotation éthique (et dans la signification usuelle attachée à ce terme), est commun à tous les peuples protestants depuis la traduction de la Bible et fait défaut à tous les autres, est une innovation de la Réforme sur un point décisif pour ma recherche» (Weber 1907-10: 322; voir aussi page 335-338) au comportement de dilettante - voire même à l'ignorance pure et simple - de son contradicteur qui «n'a pas apporté le moindre début de preuve à son hypothèse quelque peu hardie, selon laquelle j'aurais entrepris une construction idéaliste de l'histoire» (ibid: 334). Et si Weber récuse la demande de fournir des preuves statistiques c'est lorsque la demande lui paraît irréalisable ${ }^{17}$, car il ne s'y refuse pas dans d'autres occasions (ibid: 438).

\section{CONCLUSION}

La réflexion sur la preuve a permis de revenir sur le rôle de la méthode dans le contexte de la formation et de la première institutionnalisation de la sociologie au tournant des $\mathrm{XIX}^{\mathrm{e}}$ et $\mathrm{XX}^{\mathrm{e}}$ siècles.

Les rapprochements effectués ici entre Durkheim et Weber, à rebours des interprétations scolaires qui accentuent les différences, montrent que, sur nombre de points, les deux auteurs ne différaient pas significativement entre eux. Tel est le cas de l'administration statistique de la preuve mise en œuvre par Weber comme par Durkheim, sans être jamais considérée comme le seul critérium de ce qui est scientifiquement acceptable pour Durkheim comme pour Weber.

On pourra toujours penser que des différences demeurent entre l'approche de Weber et celle de Durkheim, soit en termes méthodologiques généraux - notamment en faisant usage du clivage individualisme / holisme méthodologique - ou en termes de style de la recherche mise en œuvre par l'un ou l'autre des deux auteurs et de ceux qui s'en inspirent. Des travaux, anciens ou plus récents ${ }^{18}$, ont maintenant montré combien il faut être prudent avant de chercher à dresser l'un contre l'autre ces deux styles sociologiques, tant il existe de liens entre eux en matière de méthode.

Finalement, le rapprochement porte aussi sur les aspects sociaux de la preuve. Présenter la preuve d'une assertion ou la demander revient à mettre à l'épreuve les

17 «Pour ma part, je considère comme parfaitement dérisoire l'exigence de Rachfahl, qui voudrait que fût établie ici une sorte de statistique. Ainsi doit le faire toute personne qui sait par expérience quelles difficultés inouïes s'opposent aujourd'hui à toute tentative de mesurer, concernant un objet toujours vivant, la portée d'une motivation déterminée 'relevant d'une conception du monde', même s'il ne fait aucune doute que cette motivation est présente et agissante» (Weber 1907-10: 364-5). Weber revient sur cette question plus loin pour expliquer qu'une «statistique dénombrant par exemple les calvinistes proprement dit parmi les émigrants protestant ne suffit nullement à argumenter contre l'importance de ces formes [puritaines] de vie ascétique » (ibid: 431) en raison des rapprochements qui eurent lieu entre les différentes sectes une fois celles-ci implantées sur le sol du nouveau monde.

18 On peut penser aux travaux, d'orientation si différente par ailleurs, développés par Pierre Bourdieu, Jean-Claude Chamboredon et Jean-Claude Passeron (1973) ou à ceux de Raymond Boudon (1998). 
personnes et les énoncés devant le tracé qui, symboliquement, définit qui personnes ou propositions - appartient à la 'communauté scientifique' et qui n'y appartient pas. Ces enjeux qu'ont affrontés Durkheim et Weber demeurent les mêmes pour nous: la notion de la preuve reste traversée par les enjeux scientifiques, politiques et sociaux qui animent les acteurs sociaux de la science, comme ceux qui sont tenus à la lisière de celle-ci.

\section{IRIS, Université de Paris IX-Dauphine}

\section{RÉFÉRENCES}

Bastide, Roger (1935) Eléments de sociologie religieuse, Paris: Armand Colin

Baudelot, Christian \& Establet, Roger (1984) Durkheim et le suicide, Paris: Presses universitaires de France

- (1997) «A propos des variations concomitantes», dans C-H. Cuin (ed.): 131-152

Bernard, Claude (1865) Introduction à l'étude de la médecine expérimentale, Paris: Flammarion (1966)

Berthelot, Jean-Michel (1988) «Les Règles de la méthode sociologique ou l'instauration de la méthode expérimentale en sociologie», dans E. Durkheim Les règles de la méthode sociologique, Paris: Flammarion: 7-67

Besnard, Philippe (1987) L'anomie, ses usages et ses fonctions dans la discipline sociologique depuis Durkheim, Paris: Presses universitaires de France

Borlandi, Massimo \& Mucchielli, Laurent (eds.) (1995) La sociologie et sa méthode. Les 'Règles' de Durkheim un siècle après, Paris: L'Harmattan

Boudon, Raymond (1970) L'analyse mathématique des faits sociaux, 2édition, Paris: Plon

- (1998) Etudes sur les sociologues classiques, Paris: Presses universitaires de France

Bourdieu, Pierre (1989) La noblesse d'Etat. Grandes écoles et esprit de corps, Paris: Minuit

Bourdieu, Pierre, Chamboredon, Jean-Claude \& Passeron, Jean-Claude (1973) Le métier de sociologue, $2^{\mathrm{c}}$ édition, Paris: Mouton

Cherkaoui, Mohamed (1997) «La théorie de l'explication chez Durkheim: modèle déductif-nomologique et modèle des mécanismes générateurs », dans C-H. Cuin (ed.): 153-168

Cuin, Charles-Henri (ed.) (1997) Durkheim d' un siècle à l'autre. Lectures actuelles des 'Règles de la méthode sociologique', Paris: Presses universitaires de France

Desrosières, Alain (1993) La politique des grands nombres. Histoire de la raison statistique, Paris : La découverte

Durkheim, Emile (1894) Les règles de la méthode sociologique, Paris: Presses universitaires de France (1977)

- (1897) Le suicide. Etude de sociologie, Paris: Presses universitaires de France (1976)

- (1904-5) L'évolution pédagogique en France, Paris: Presses universitaires de France (1969)

- (1998) Correspondance avec Marcel Mauss, Paris: Presses universitaires de France

Fauconnet, Paul \& Mauss, Marcel (1901) «Sociologie», dans M. Mauss (1969) CEuvres, Paris: Minuit, vol.3: 139-177

Fréchet, Maurice \& Halbwachs, Maurice (1924) Le calcul des probabilités à la portée de tous, Paris: Dunod

Ghosh, Peter (2003) «Max Weber's idea of 'Puritanism': a case study in the empirical construction of the Protestant ethic », History of European Ideas, 29(2): 183-222

Grossein, Jean-Pierre (2000) «Présentation», dans M. Weber (1915) Confucianisme et taoïsme, trad. française, Paris: Gallimard: i-xxv 
Halbwachs, Maurice (1912) La classe ouvrière et les niveaux de vie, Paris \& New York: Gordon and Breach (1970)

- (1913) La théorie de l'homme moyen. Essai sur Quetelet et la statistique morale, Paris: Alcan

- (1923) «L'expérimentation statistique et les probabilités», dans M. Halbwachs (1972) Classes sociales et morphologie, Paris : Minuit: 275-307

- (1925) «Les origines puritaines du capitalisme», Revue d' histoire et de philosophie religieuse, 5 : 132-154

- (1929) «Max Weber: un homme, une œuvre», Annales, 1: 81-88

- (1930) Les causes du suicide, Paris: Alcan

- $\quad$ (1933) «La loi en sociologie», dans M. Halbwachs (1972) Classes sociales et morphologie, Paris: Minuit: 308-328

- (1935) «La statistique en sociologie», dans M. Halbwachs (1972) Classes sociales et morphologie, Paris : Minuit: 329-348

Hedström, Peter \& Swedberg, Richard (eds.) (1998) Social Mechanisms, Cambridge: Cambridge university Press

Lukes, Steven (1973) Emile Durkheim: His Life and Work. A Historical and Critical Study, London: Penguin Press

Mauss, Marcel (1927) «Note de méthode sur l'extension de la sociologie», dans M. Mauss (1969) Euvres, vol. 3, Paris: Minuit: 283-297

- (1934) «Fragment d'un plan de sociologie générale descriptive», dans M. Mauss (1969) Euvres, vol.3, Paris: Minuit: 303-364

Pareto, Vilfredo (1898) «Le suicide», dans Mythes et idéologies, Euvres complètes de Pareto, vol. 6, Genève: Droz: 122-124

Pope, William (1976) Durkheim's «Suicide».A Classic Analyzed, Chicago: Chicago university Press

Schmauss, Warren (2002) «Epistemology and philosophy of science», dans W. Pickering (ed.) Durkheim Today, Oxford: Berghahn Books: 39-54

Simiand, François (1907) Le salaire des ouvriers des mines de charbon en France. Contribution à la théorie économique du salaire, Paris: Cornély

- (1922) Statistiques et expérience. Remarques de méthode, Paris : Rivière

- (1932) Le salaire, l'évolution sociale et la monnaie, Paris: Alcan

Steiner, Philippe (1992) «L'Année sociologique et la réception de l'œuvre de Max Weber», Archives européennes de sociologie, 33(2): 329-349

- $\quad$ (2000) «Crise, effervescence sociale et socialisation», dans M. Borlandi \& M. Cherkaoui (eds.) Le suicide un siècle après Durkheim, Paris: Presses universitaires de France: 63-85

- (2003) Economie et religion. Principes de la sociologie économique durkheimienne, à paraître

Stigler, Stephen M. (1986) The History of Statistics. The Measurement of Uncertainty before 1900, Cambridge Mass. : Harvard university Press (2000)

Weber, Max (1907-1910) «Anticritiques», dans M. Weber (2003) L'éthique protestante et l' esprit du capitalisme, trad. française, Paris: Gallimard: 319-446

- (1908) «Sulla psicologia del lavoro industriale», trad. italienne dans M. Weber (1983) Metodo e ricerca nella grande industria, Milano: Franco Angeli : 121-291

- (1919) «La profession et la vocation de savant», dans M. Weber (2003) Le savant et le politique, trad. française, Paris: La découverte: 67-110

- (1921) Economie et société, trad. française, Paris: Plon (1972)

Westergaard, Harald (1932) Contribution to the History of Statistics, New York: Kelley (1969) 